\title{
Letter to the editor: SARS-CoV-2 detection by real-time RT-PCR
}

Trestan Pillonel ${ }^{1}$, Valentin Scherz ${ }^{1}$, Katia Jaton ${ }^{1}$, Gilbert Greub ${ }^{1}$, Claire Bertelli ${ }^{1}$

1. Institute of Microbiology, Lausanne University Hospital and University of Lausanne, Lausanne, Switzerland

Correspondence: Claire Bertelli (Claire.Bertelli@chuv.ch)

Citation style for this article:

Pillonel Trestan, Scherz Valentin, Jaton Katia, Greub Gilbert, Bertelli Claire . Letter to the editor: SARS-CoV-2 detection by real-time RT-PCR. Euro Surveill. 2020;25(21): pii=2000880. https://doi.org/10.2807/1560-7917.ES.2020.25.21.2000880

To the editor: The rapid development of open diagnostic methods for the detection of severe acute respiratory syndrome coronavirus 2 (SARS-CoV-2) has been key to build capacity for efficient molecular diagnostic tests in laboratories worldwide. These methods based on real-time RT-PCR were recognised as reference protocols since mid-January, at the onset of the pandemic in China, and relayed - although not validated - by the World Health Organization [1]. We would first like to acknowledge the contribution of Corman et al. [2] who were among the first to provide primers and probes for three genes ( $E, N$ and RdRp), that have been widely implemented across the globe to tackle the coronavirus disease (COVID-19) pandemic. They recommended the use of the PCR targeting the $E$ gene followed by confirmation with RdRp primers combined to a SARSCoV-2 specific probe.

Our diagnostic laboratory also implemented and used the above-mentioned assays [2] throughout the first months of the pandemic on its automated platform [3]. RNA was extracted from clinical samples with the MagNA Pure 96 System (Roche, Basel, Switzerland) and the real-time reverse-transcription PCR (RT-PCR) was performed on a QuantStudio 7 system (Applied Biosystems, Waltham, United States). RT-PCRs targeting $E$ and RdRp were used routinely in parallel for 893 samples. In 115 cases with positive amplification by both RT-PCRs, the RdRp assay showed a significantly (paired Wilcoxon rank test, $p$ value < o.001) higher average cycle threshold (CT) (25.0 CT, interquartile range (IQR): 24.6-27.9) than the E target (22.6 CT, IQR: 19.3-25.8). Furthermore, positive $E$ and negative RdRp results were obtained in 10 cases $(1 \%)$, triggering further investigations, since patients were unlikely to be infected by other SARS-related viruses that can also be amplified in the E assay. Conversely, no negative $E$ and positive RdRp RT-PCR results were observed.

After careful review of the initial manuscript and analysis of SARS-CoV-2 and other coronavirus sequences, it appeared that the proposed RdRp reverse primer contained an incorrect degenerate base (S), that does not match with the SARS-CoV-2 RNA sequence, as shown in the alignment of Corman et al. Figure 2 [2]. Indeed, the proposed RdRp_SARSr-R sequence (CARATGTTAAASACACTATTAGCATA, $\mathrm{R}=[\mathrm{AG}], \mathrm{S}=[\mathrm{GC}]$ ) does not match any of the 1,623 SARS-CoV-2 complete genome sequences publicly available in the National Center for Biotechnology Information (NCBI) database as at 7 May 2020. Instead, the corrected RdRp_SARSr-R2 sequence (CARATGTTAAARACACTATTAGCATA, $R=[A G]$ ) should allow to amplify SARS-CoV-2 genetic material, including loosely related bat and human sequences, with improved efficiency. Two additional nucleotides in the pan-Sarbecovirus probe RdRP_SARSr-P1 (CCAGGTGGWACRTCATCMGGTGATGC, $\mathrm{W}=[\mathrm{AT}], \mathrm{R}=$ [AG], $M=[A C]$ ) should also be replaced (RdRP_SARSrP1b, CCAGGTGGWACMTCATCMGGWGATGC, $M=[A C]$, $W=[A T])$ to improve similarity with the SARS-CoV-2 and bat coronavirus genetic sequence while retaining the pan-Sarbecovirus compatibility. These observations based on in silico alignments should be confirmed by wet-laboratory experiments, but they could explain the lower sensitivity of the RdRp RT-PCR also shown by Vogels et al. [4] and point towards potential improvements.

As the pandemic spreads, many laboratories worldwide, including in low-resource countries that may not rely on expensive commercial kits, implement routine diagnostic tests. Thus, we think that such information is critical to ensure a proper detection of SARS-CoV-2 infections, allowing efficient isolation and preventing further transmission of the virus.

Acknowledgements

We gratefully acknowledge the work of all technicians who performed the molecular diagnostic of SARS-CoV-2 at Lausanne University Hospital during the past months. 
None declared.

\section{Authors' contributions}

Conceived and designed the manuscript: $C B$, VS, TP, GG. Contributed to the analysis: CB, TP, VS. Implemented and supervised the molecular diagnostic tests: KJ, GG. Drafted the manuscript: CB. Reviewed and approved the manuscript: all authors.

\section{References}

1. World Health Organization (WHO). Molecular assays to diagnose COVID-19. Geneva: WHO; 24 Jan 2020. Available from: https://www.who.int/who-documents-detail/molecularassays-to-diagnose-covid-19-summary-table-of-availableprotocols

2. Corman VM, Landt O, Kaiser M, Molenkamp R, Meijer A, Chu DK, et al. Detection of 2019 novel coronavirus (2019-nCoV) by real-time RT-PCR. Euro Surveill. 2020;25(3):2000045. https:// doi.org/10.2807/1560-7917.ES.2020.25.3.2000045 PMID: 31992387

3. Greub G, Sahli R, Brouillet R, Jaton K. Ten years of R\&D and full automation in molecular diagnosis. Future Microbiol. 2016;11(3):403-25. https://doi.org/10.2217/fmb.15.152 PMID: 27028061

4. Vogels CBF, Brito AF, Wyllie AL, Fauver JR, Ott IM, et al. Analytical sensitivity and efficiency comparisons of SARS-COV-2 qRT-PCR primer-probe sets. medRxiv 2020;2020.03.30.20048108; (Preprint). https://doi. org/10.1101/2020.03.30.20048108

\section{License, supplementary material and copyright}

This is an open-access article distributed under the terms of the Creative Commons Attribution (CC BY 4.0) Licence. You may share and adapt the material, but must give appropriate credit to the source, provide a link to the licence and indicate if changes were made.

Any supplementary material referenced in the article can be found in the online version.

This article is copyright of the authors or their affiliated institutions, 2020. 